\title{
Alumina Thin-Film Deposition on Rough Topographies Comprised of Vertically Aligned Carbon Nanotubes: Implications for Mem-branes, Sensors, and Electrodes
}

Lev Rovinsky,*, Barun Kumar Barick², Elnatan Lieberman, Efrat Shawat Avraham³, Gilbert Daniel Nessim³, Tamar Segal-Peretz ${ }^{2}$ and Noa Lachman ${ }^{1, *}$

${ }^{1}$ Department of Materials Science and Engineering, Tel Aviv University, 69978, Israel

${ }^{2}$ Department of Chemical Engineering, Technion, Haifa, 3200003, Israel

3Department of Chemistry, Bar-Ilan University, 5290002, Israel

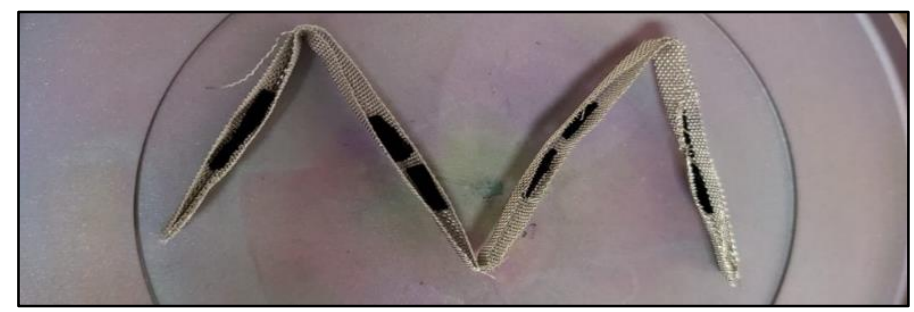

Figure S-1. - Custom jig made from $\mathrm{SS}_{304}$ stainless steel mesh 4oLPI, folded into freestanding trench-line structure. The large gray circles are the ALD apparatus chamber floor. Thus, arrays are positioned perpendicularly to chamber floor and along precursor gas flow. The arrays visible here are pristine.

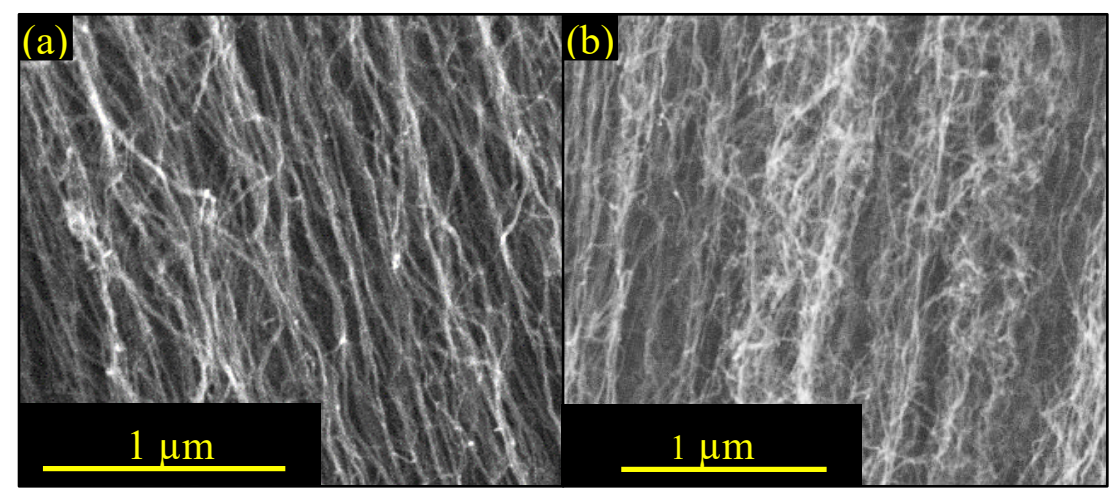

Figure S-2. - Comparison between the same relative location on a pristine sample (a) and a sample that was functionalized at $475^{\circ} \mathrm{C}$ for one minute (b).

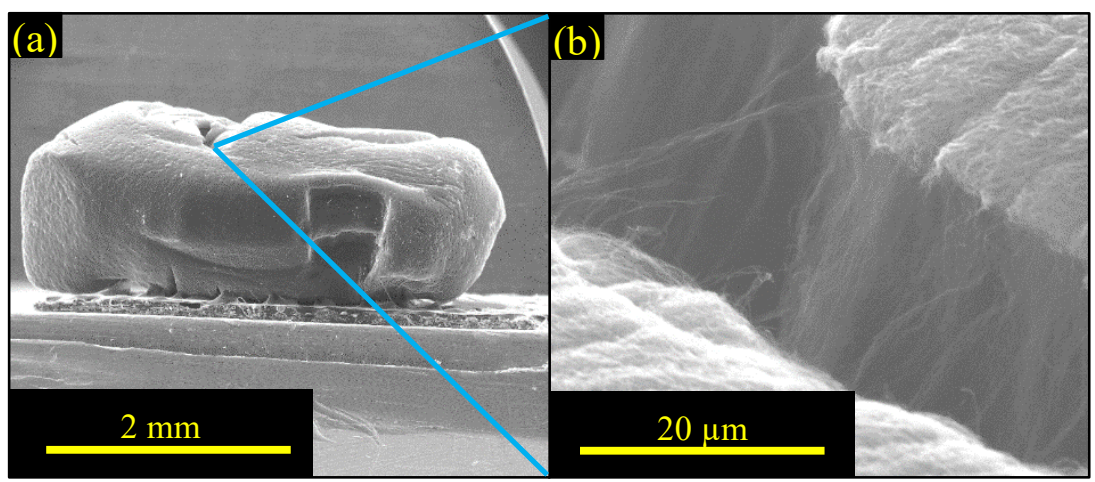

Figure S-3. - An array that underwent functionalization at exceedingly rapid heating rate of $5{ }^{\circ} \mathrm{C} / \mathrm{min}$. On the left, the residue of what was once $10 \mathrm{~mm}$ wide (a). On the right, inside (a), several CNT-like structures are visible (b). 

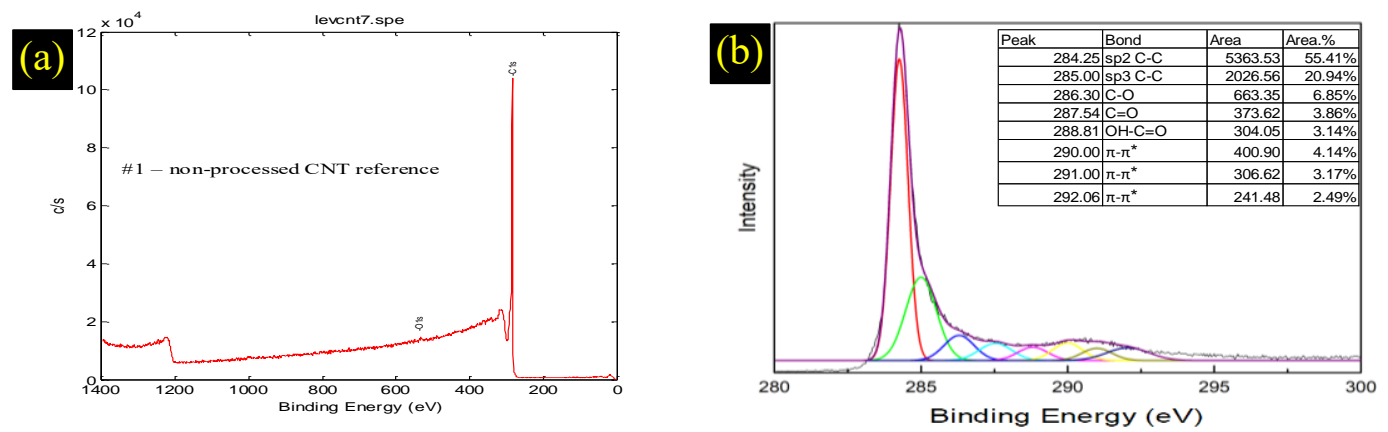

Figure S-4. - XPS analysis of pristine VACNT array, sample center. (a) Complete survey (b) C1s with fitted curves and their peak areas (inset in (b)).
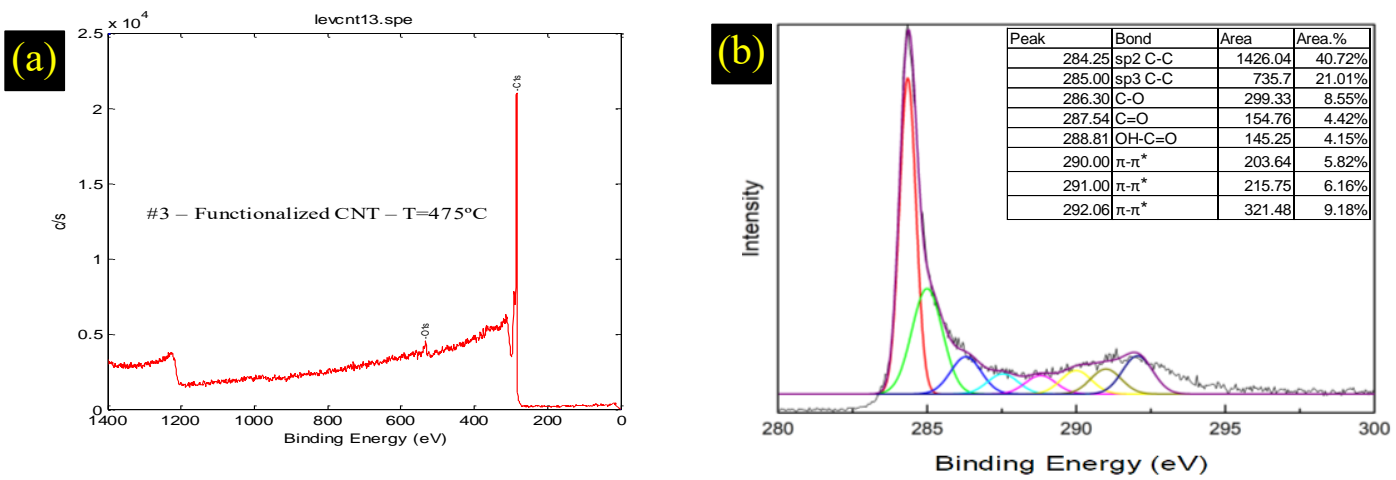

Figure S-5. - XPS analysis of VACNT, functionalized at $475{ }^{\circ} \mathrm{C}$, for $1 \mathrm{~min}$, sample center. (a) Complete survey (b) C1s with fitted curves and their peak areas (inset in (b)).
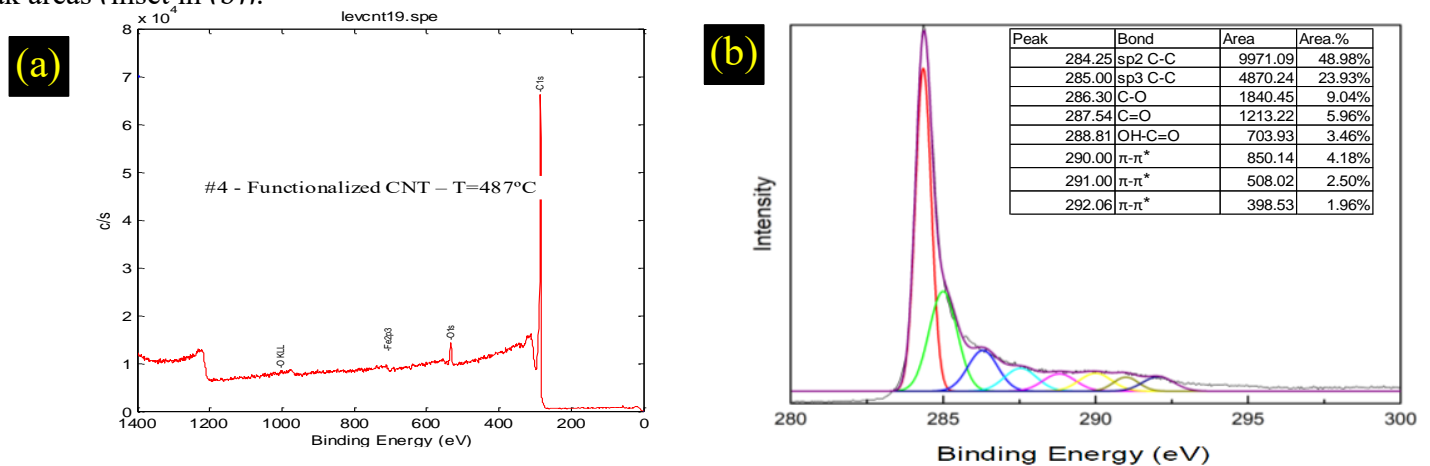

Figure S-6. - XPS analysis of VACNT, functionalized at $487^{\circ} \mathrm{C}$, for $1 \mathrm{~min}$, sample center. (a) Complete survey (b) C1s with fitted curves and their peak areas (inset in (b)).
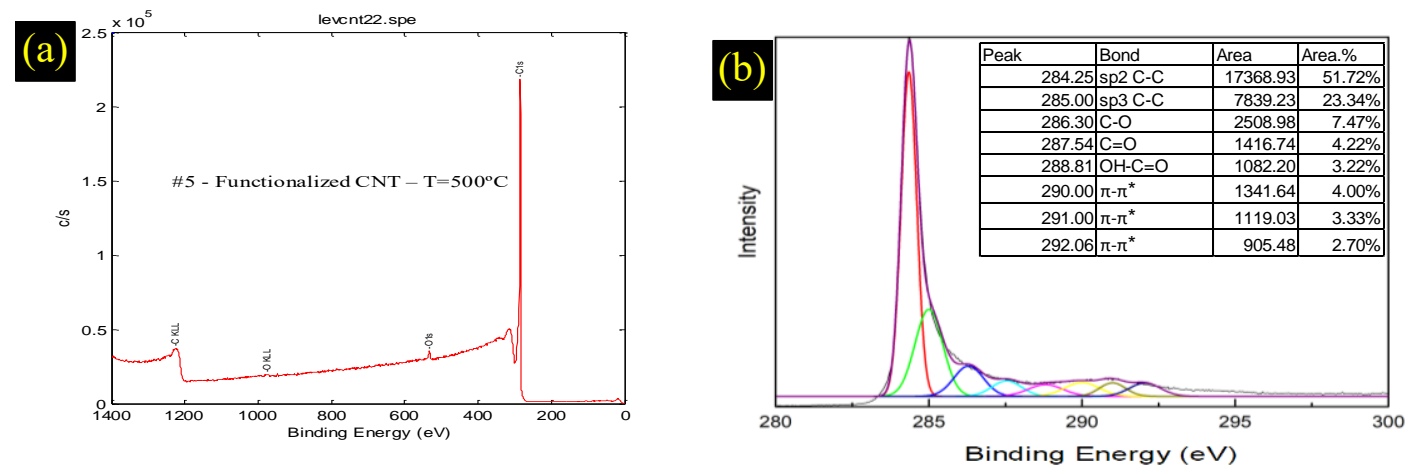

Figure S-7. - XPS analysis of VACNT, functionalized at $500{ }^{\circ} \mathrm{C}$, for $1 \mathrm{~min}$, sample center. (a) Complete survey (b) C1s with fitted curves and their peak areas (inset in (b)). 

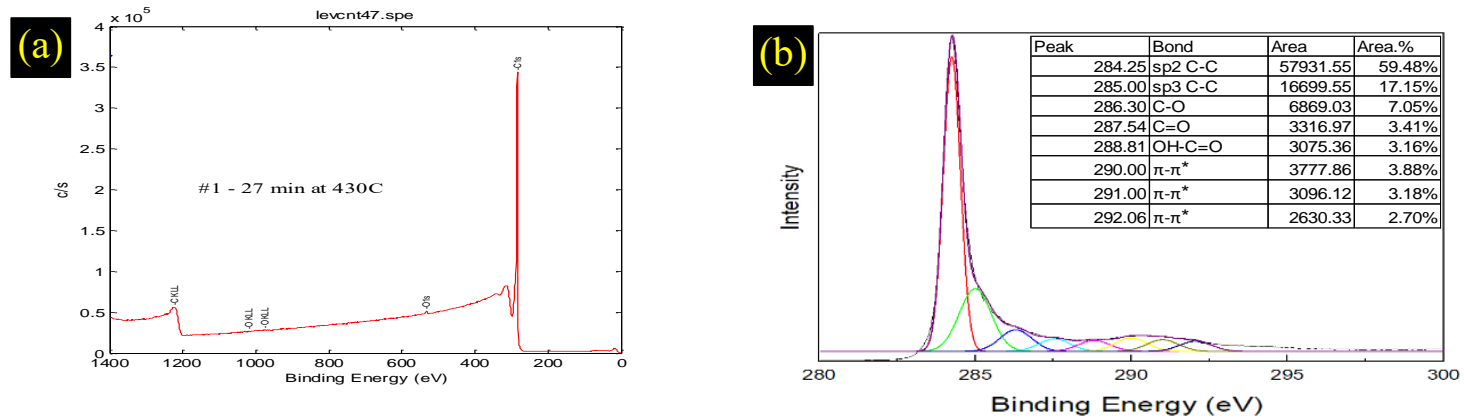

Figure S-8. - XPS analysis of VACNT, functionalized at $430^{\circ} \mathrm{C}$, for $20 \mathrm{~min}$, sample center. (a) Complete survey (b) C1s with fitted curves and their peak areas (inset in (b)).
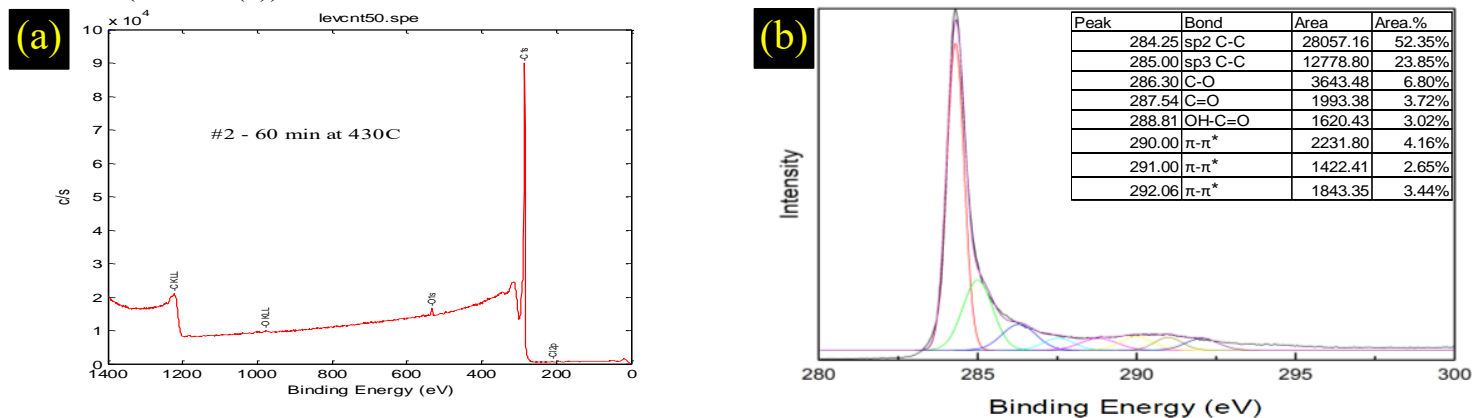

Figure S-9. - XPS analysis of VACNT, functionalized at $430{ }^{\circ} \mathrm{C}$, for $60 \mathrm{~min}$, sample center. (a) Complete survey (b) C1s with fitted curves and their peak areas (inset in (b)).
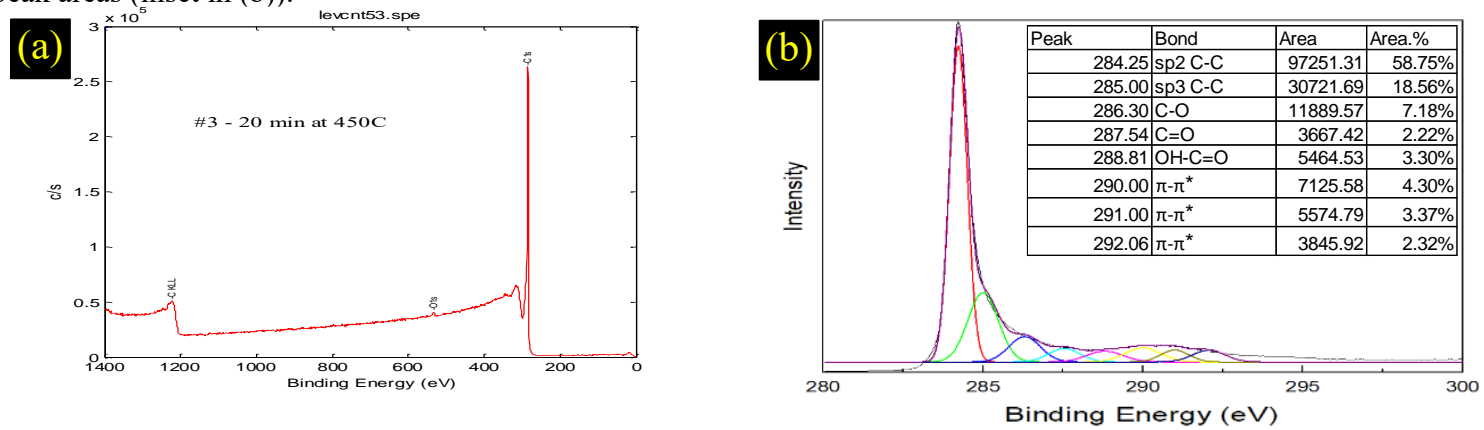

Figure S-10. - XPS analysis of VACNT, functionalized at $450{ }^{\circ} \mathrm{C}$, for $20 \mathrm{~min}$, sample center. (a) Complete survey (b) C1s with fitted curves and their peak areas (inset in (b)).
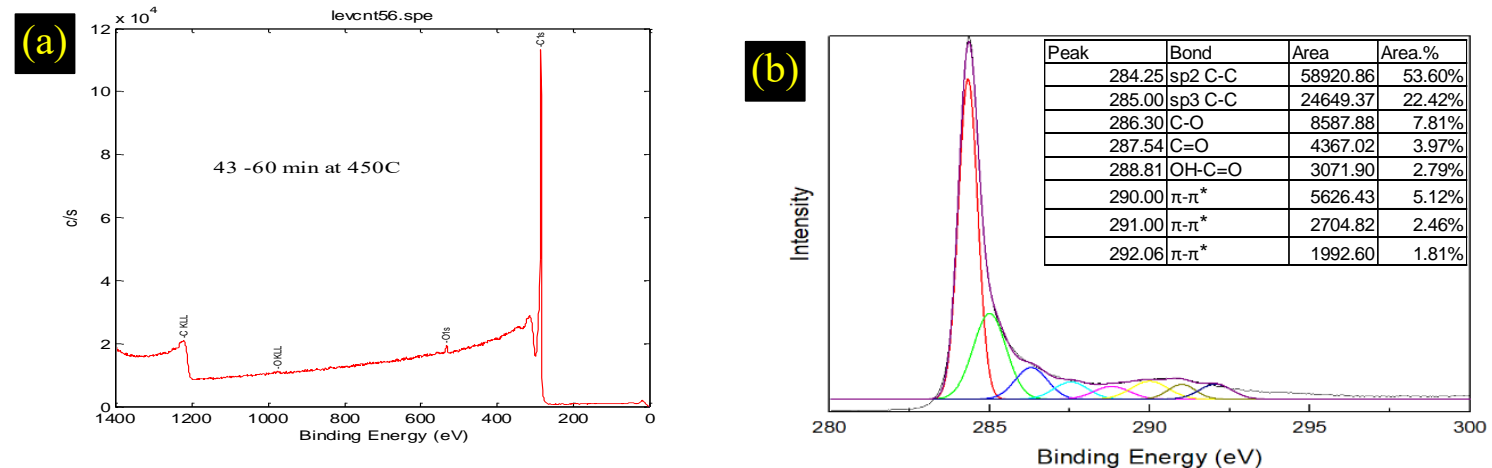

Figure S-11. - XPS analysis of VACNT, functionalized at $450{ }^{\circ} \mathrm{C}$, for $60 \mathrm{~min}$, sample center. (a) Complete survey (b) C1s with fitted curves and their peak areas (inset in (b)). 

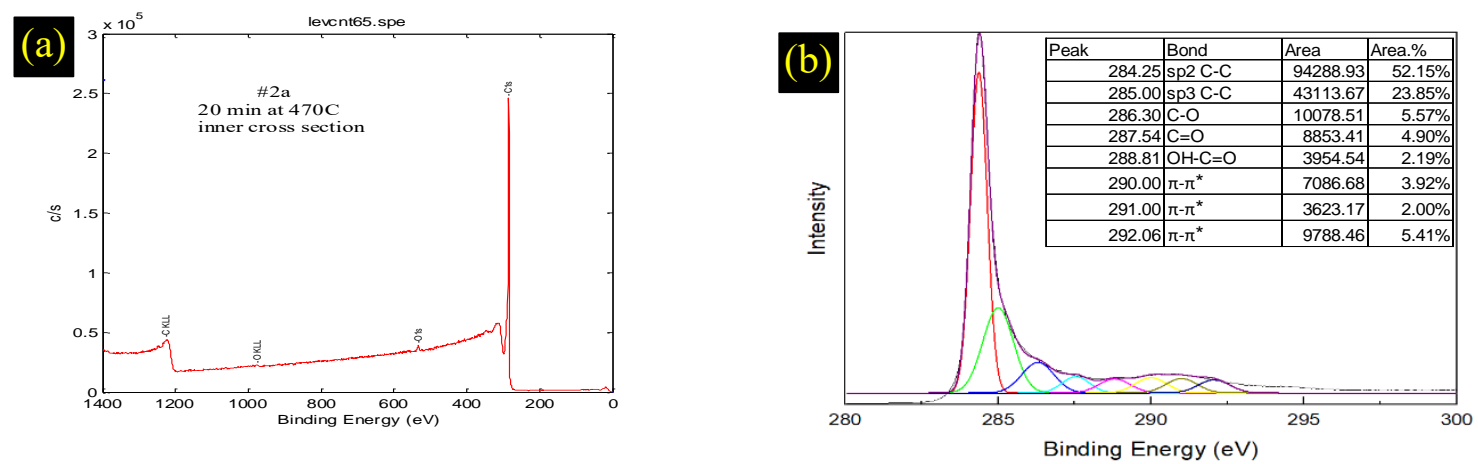

Figure S-12. - XPS analysis of VACNT, functionalized at $470{ }^{\circ} \mathrm{C}$, for $20 \mathrm{~min}$, sample center. (a) Complete survey (b) C1s with fitted curves and their peak areas (inset in (b)).
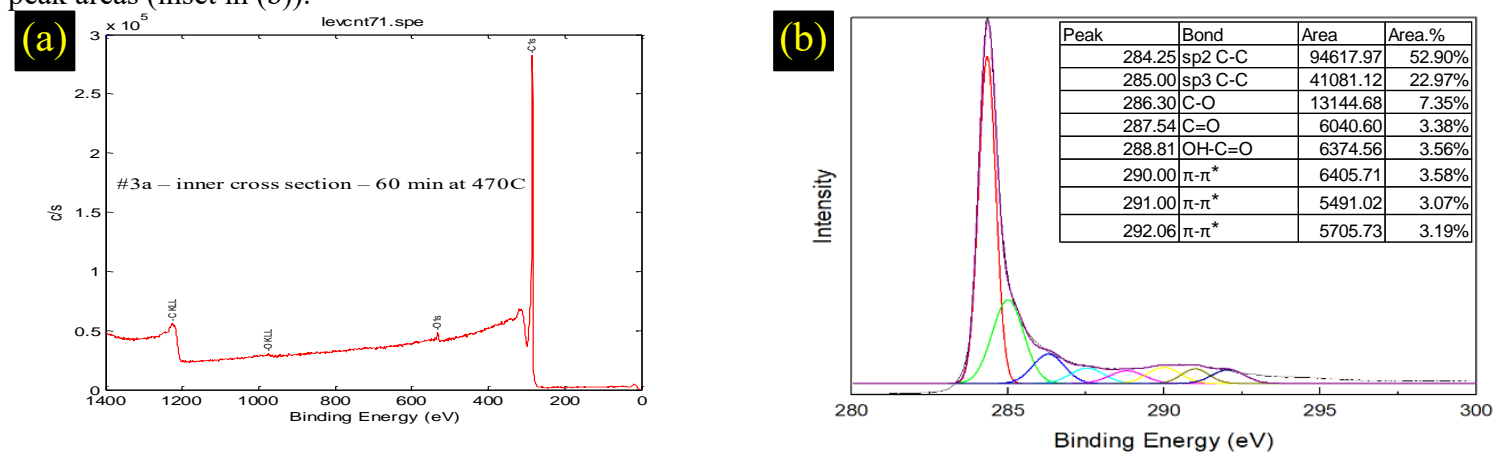

Figure S-13. - XPS analysis of VACNT, functionalized at $470{ }^{\circ} \mathrm{C}$, for $60 \mathrm{~min}$, sample center. (a) Complete survey (b) $\mathrm{C} 1 \mathrm{~s}$ with fitted curves and their peak areas (inset in (b)).
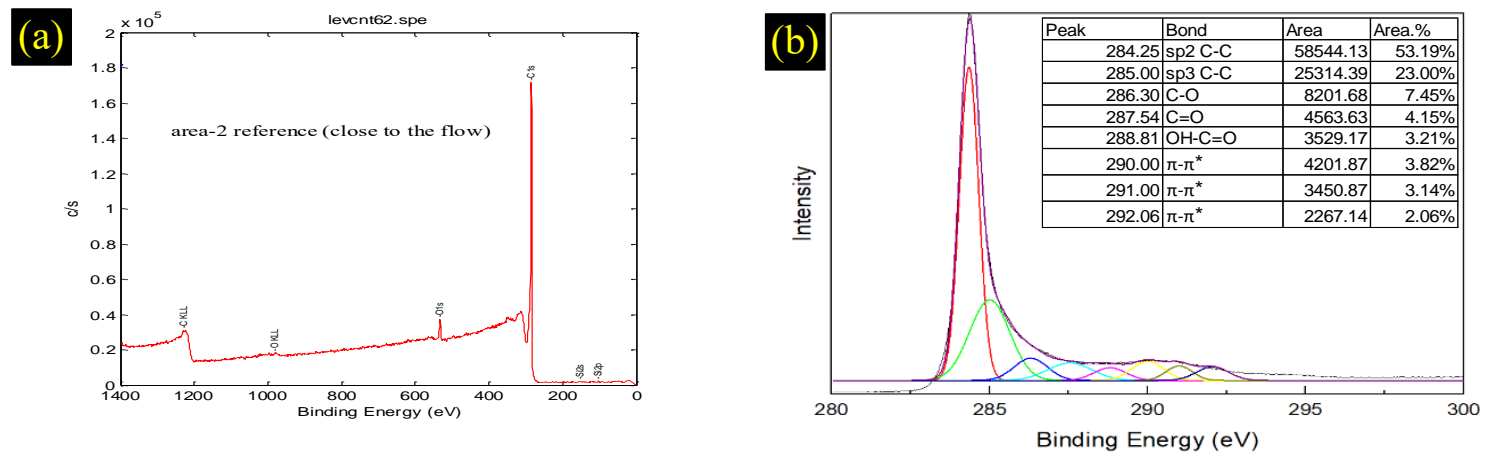

Figure S-14. - XPS analysis of pristine VACNT, sample periphery. (a) Complete survey (b) C1s with fitted curves and their peak areas (inset in (b)).
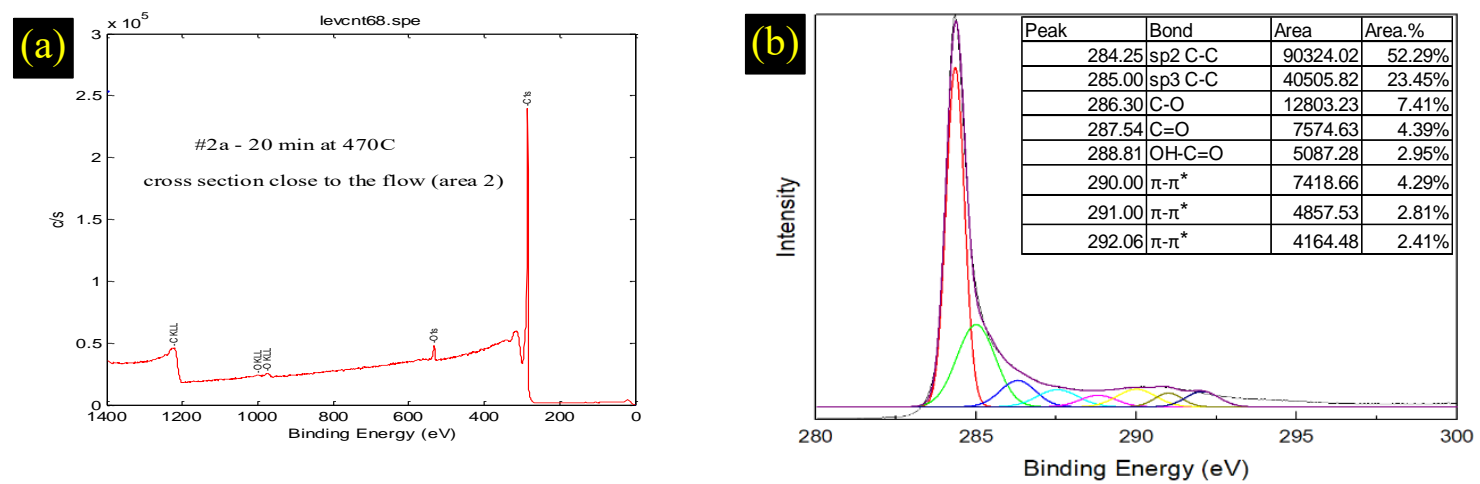

Figure S-15. - XPS analysis of VACNT, functionalized at $470{ }^{\circ} \mathrm{C}$, for $20 \mathrm{~min}$, sample periphery. (a) Complete survey (b) C1s with fitted curves and their peak areas (inset in (b)). 
(a)

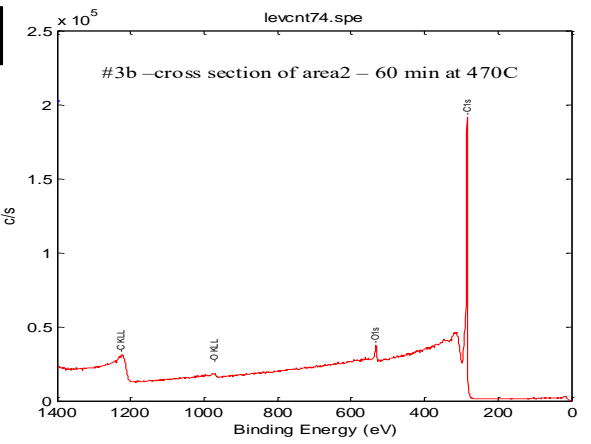

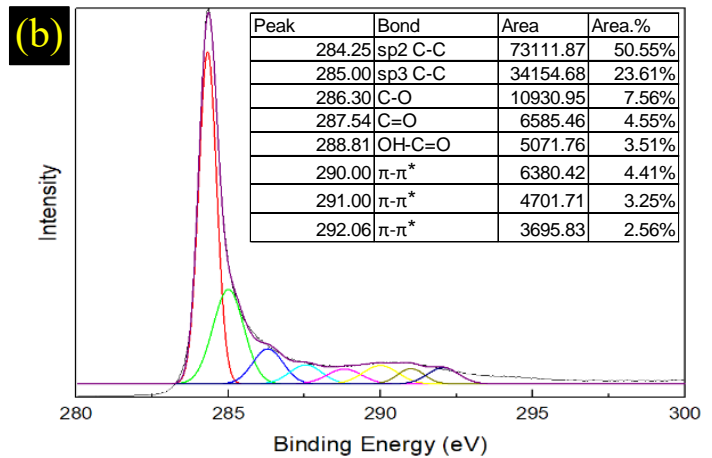

Figure S-16. - XPS analysis of VACNT, functionalized at $470{ }^{\circ} \mathrm{C}$, for $60 \mathrm{~min}$, sample periphery. (a) Complete survey (b) C1s with fitted curves and their peak areas (inset in (b)).

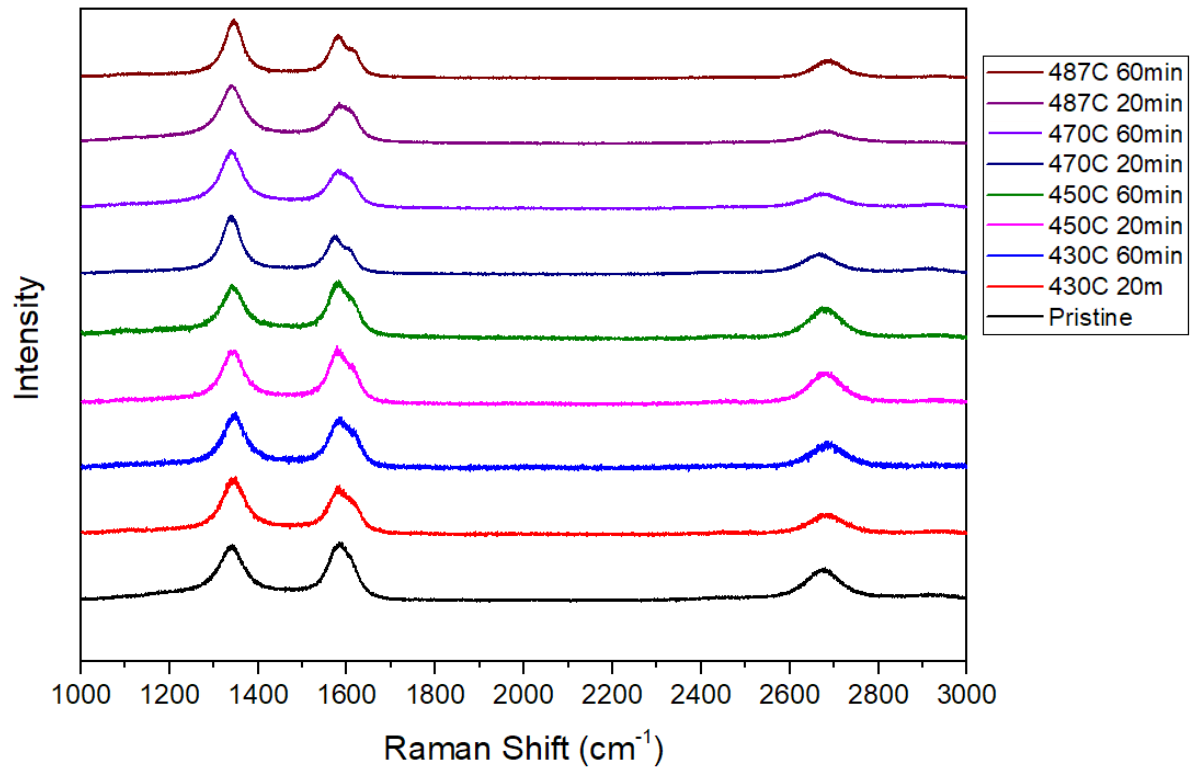

Figure S-17. - Raman spectroscopy curves for several functionalized samples.
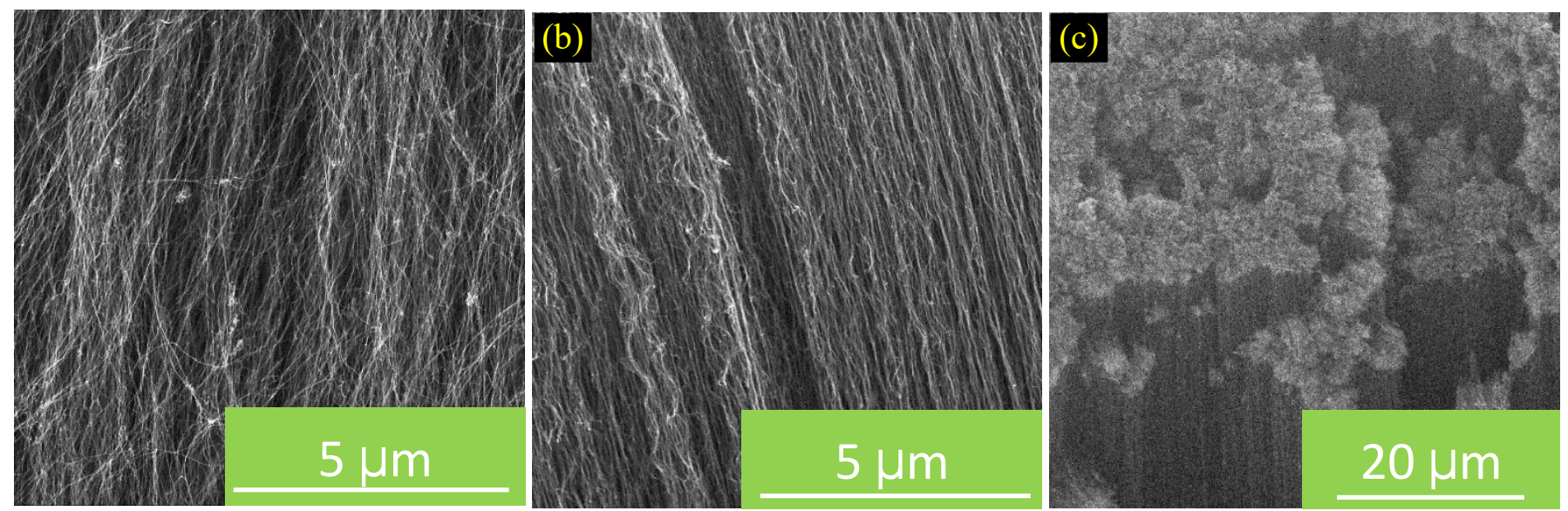

Figure S-18 - Regions of morphology with different waviness found throughout the VACNT array. Each of the three zones is formed under different circumstances, experiencing different forces during growth, thus creating different morphologies. The ultimate structure is a relatively straight center (b) located between a fuzz bottom (a) and amorphous carbon on top (c). 

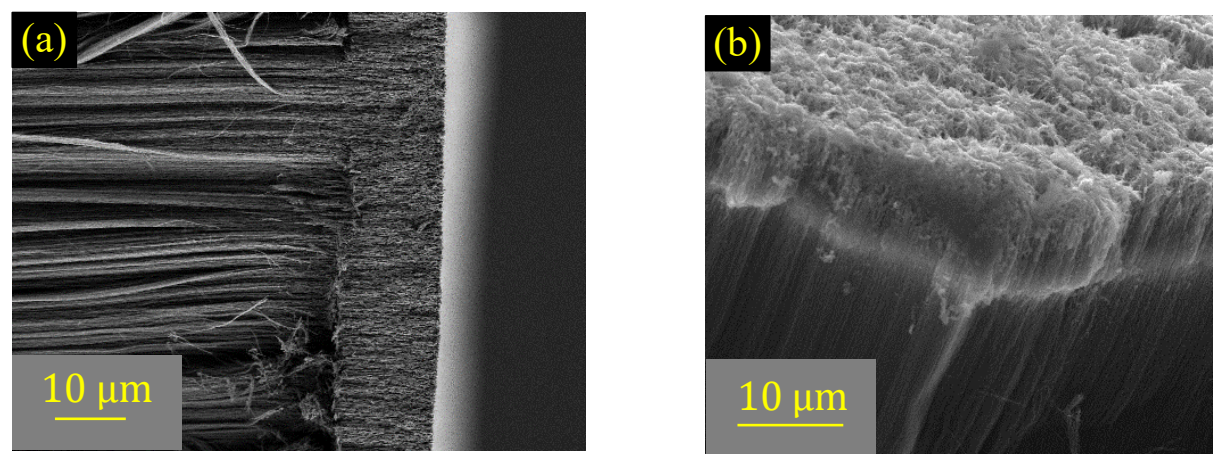

Figure S-19. - SEM images showing nanocomposite materials of functionalized VACNT and $\mathrm{Al}_{2} \mathrm{O}_{3}$ (functionalization: 1 hour at $470^{\circ} \mathrm{C}$ ) coated using different precursor pulse lengths: (a) showing $15 \mu \mathrm{m}$ suffocation depth at $200 \mathrm{~ms}$ pulse and (b) showing suffocating at $10 \mu \mathrm{m}$ at $150 \mathrm{~ms}$ pulse.

\begin{tabular}{|c|c|c|c|}
\hline $\begin{array}{l}\text { ALD parameters } \\
\left.\text { [TMA: } \mathrm{N}_{2}: \mathrm{H}_{2} \mathrm{O}: \mathrm{N}_{2}\right] \\
\text { [sec:sec:sec:sec] }\end{array}$ & $\begin{array}{c}\text { ALD } \\
\text { cycles } \\
\text { [quantity] }\end{array}$ & $\begin{array}{c}\text { ALD } \\
\text { Temp. } \\
{\left[{ }^{\circ} \mathrm{C}\right]}\end{array}$ & $\begin{array}{c}\text { Penetration Depth } \\
{[\mu \mathrm{m}]}\end{array}$ \\
\hline 30:30:30:30 & 500 & 100 & 20 \\
\hline 6o:6o:6o:6o & 250 & 100 & 142 \\
\hline 90:90:90:90 & 250 & 100 & 90 \\
\hline 120:120:120:120 & 250 & 100 & 40 \\
\hline 120:90:120:90 & 250 & 100 & 110 \\
\hline 120:30:120:30 & 250 & 100 & 78 \\
\hline 180:30:180:30 & 250 & 100 & 73 \\
\hline 6o:6o:6o:6o (jig) & 250 & 100 & 200 \\
\hline $60: 60: 60: 60^{(H T)}$ & 250 & 200 & 350 \\
\hline
\end{tabular}

\section{Table ST-1. - Contents of figure 5.}

"jig" represents the application of ALD stand.

"HT" represents the use of high temperature, $200^{\circ} \mathrm{C}$, compared to $100^{\circ} \mathrm{C}$. 